\title{
Purpureocillium lilacinum (Hypocreales: Ophiocordycipitaceae) como biocontrolador de Nacobbus aberrans (Tylenchida: Pratylenchidae) y Meloidogyne incognita (Tylenchida: Meloidogynidae) en tomate cv. Río Grande
}

Purpureocillium lilacinum (Hypocreales: Ophiocordycipitaceae) as a biocontrol agent of Nacobbus aberrans (Tylenchida: Pratylenchidae) and Meloidogyne incognita (Tylenchida: Meloidogynidae) in tomato cv. Río Grande

\section{Rómulo García-Velasco ${ }^{1}$ [D , Edgar Andrés Chavarro-Carrero ${ }^{1}$}

${ }^{1}$ Centro Universitario Tenancingo, Universidad Autónoma del Estado de México, km 1.5 Carretera Tenancingo-Villa Guerrero, 52400, Estado de México, México. *Autor para correspondencia: rgarciave@uaemex.mx

Fecha de recepción:

26 de abril de 2020

Fecha de aceptación:

30 de julio de 2020

\section{Disponible en línea:}

5 de noviembre de 2020

Este es un artículo en acceso abierto que se distribuye de acuerdo a los términos de la licencia Creative Commons.

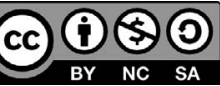

Reconocimiento-

NoComercial-

CompartirIgual 4.0

Internacional

\section{RESUMEN}

Dentro de los problemas fitosanitarios más importantes del tomate en México, se registra el daño de nematodos agalladores, como el ocasionado por Nacobbus aberrans (Thorne) Thorne y Allen y Meloidogyne incognita (Kofoid y White) Chitw. El manejo tradicional consiste en la aplicación de nematicidas sintéticos; sin embargo, la problemática ambiental asociada a estos productos impulsa el estudio de alternativas nuevas. En ese sentido, se evaluó el control de tres productos formulados con Purpureocillium lilacinum y del nematicida fluensulfone contra $N$. aberrans y $M$. incognita en plantas de tomate. Nemaroot ${ }^{\circledast}$, BioAct Prime ${ }^{\circledR}$, Nematicida PI ${ }^{\oplus}$ y Nimitz 480 EC $^{\circledast}$ fueron aplicados a dosis comercial; asimismo, se incluyeron controles inoculados con N. aberrans o M. incognita sin tratar y un control sin inoculaciones. El diseño experimental fue completamente al azar, con siete tratamientos y diez repeticiones. Se evaluó el índice de agallamiento (IA), así como el número de masas de huevos (NMH), los huevos por gramo de raíz (NHG) y la efectividad biológica (EB). Los bionematicidas redujeron drásticamente el IA, NMH y NHG; de igual manera, el nivel de control fue mayor a medida que se incrementó la concentración de esporas en el producto, lo cual se reflejó en la EB en un rango de 76.6-90.1\% para N. aberrans y 77.2-92.4\% en M. incognita; en este mismo orden, con fluensulfone fueron de 61.7 y $65.5 \%$.

\section{PALABRAS CLAVE}

Nematodos agalladores, nematicidas, bionematicidas, índice de agallamiento, efectividad biológica.

\section{ABSTRACT}

Among the most important phytosanitary problems of tomato in Mexico is the damage of root-knot nematodes, such as that caused by Nacobbus aberrans (Thorne) Thorne \& Allen, and Meloidogyne incognita (Kofoid y White) Chitw. Traditional management consists of the application of synthetic nematicides. However, environmental problems associated with these products make the study of new alternatives necessary. We evaluated the level of control provided by three products formulated with Purpureocillium lilacinum and the nematicide fluensulfone against $N$. aberrans and M. incognita in tomato seedlings. Nemaroot ${ }^{\circledR}$, BioAct Prime $^{\circledR}, \mathrm{Nematicida} \mathrm{PI}^{\circledR}$ and Nimitz $480 \mathrm{EC}^{\circledR}$ were applied at commercial dose. We also included inoculated control treatments with $N$. aberrans or untreated $M$. incognita, as well as a control without nematodes and without treatment. The experimental design was completely randomized with seven treatments and ten repetitions. We evaluated the gall index (IA), number of egg masses (NMH), number of eggs per root gram (NHG), and biological effectiveness (EB). The bionematicides drastically reduced the IA, NMH and NHG; the control level was greater as the concentration of spores in the product increased, which reflected in the EB at a range of 76.6-90.1\% for N. aberrans and 77.2-92.4 $\%$ in M. incognita; in this same order with fluensulfone, it was 61.7 and $65.5 \%$.

\section{KEYWORDS}

Root-knot nematodes, nematicides, bionematicides, gall index, biological effectiveness. 


\section{INTRODUCCIÓN}

El cultivo de tomate (Solanum lycopersicum L.) es uno de los más importantes en México; a nivel nacional, en 2019 (riego + temporal) se reportó una superficie sembrada de 47,151 ha y una producción de 3,221,557 t (SIAP 2020). Sin embargo, este cultivo presenta varios problemas fitosanitarios, como el ocasionado por Nacobbus aberrans (Thorne) Thorne y Allen y Meloidogyne incognita (Kofoid y White) Chitwood (Cepeda-Siller et al. 2018; Dahlin et al. 2019). Para su control, se utilizan tradicionalmente nematicidas sintéticos, los cuales generan un impacto ambiental negativo, así como un alto costo (Huang et al. 2018).

Recientemente, se han desarrollado moléculas nuevas como el fluopyram y el fluensulfone con buen control de nematodos agalladores y bajos niveles ecotóxicos (Dahlin et al. 2019). Esta segunda molécula en condiciones in vitro ha demostrado su control contra juveniles J2 de N. aberrans de hasta 100\% de mortalidad, a una concentración de 288 ppm (Cabrera et al. 2014). En plantas de pimientos infectadas con $M$. incognita, la efectividad defluensulfoneha sido comprobada mediante aplicaciones foliares, aunque las aplicaciones al suelo no resultaron efectivas para el control de este nematodo (Oka et al. 2012). Fluensulfone es una molécula con un modo de acción nematicida, con efectos pleiotrópicos dentro de las cuales se encuentra la inhibición tanto del crecimiento, como de la oviposición de huevos, de la eclosión, de la alimentación y de la locomoción (Kearn et al. 2014). En cambio, fluopyram no tiene un modo de acción claro, pues actúa como un producto nematistático (Faske y Hurd 2015), y reduce la eclosión de huevos, así como la motilidad de $\mathrm{J} 2$ y la penetración de la raíz del juvenil J2 (Beeman y Tylka 2018); también actúa en el sistema respiratorio celular, al inhibir la succinato coenzima Q reductasa (Bayer CropScience 2020).

Cabe señalar que el uso de productos biológicos en la agricultura representa una alternativa para la producción sostenible de alimentos sin afectar el ambiente (Companioni et al. 2019). En este sentido, Purpureocillium lilacinum (Thom) Luangsa-ard, Houbraken, Hywel-Jones \& Samson, 2011 [Basiónimo = Penicillium lilacinum (Thom) Samson] (Luangsa-Ard et al. 2011), en diferentes investigaciones in vitro e in vivo, ha mostrado ser un eficiente hongo nematófago (Dahlin et al. 2019; Gortari y Hours 2016; Núñez-Camargo et al. 2012). Los efectos de filtrados de P. lilacinus fueron tóxicos para especies pertenecientes a los géneros Meloidogyne y Heterodera, con la inducción de parálisis de J2 de M. incognita (Cayrol et al. 1989). De igual forma, Kiewnick y Sikora (2006) reportaron en P. lilacinus cepa 251 una reducción del índice de agallamiento de 66\% de $M$. incognita, un número de masas de huevos de $74 \%$ y una población final en la raíz de $71 \%$ menos, en comparación con el control inoculado. Un estudio más reciente de Purpureocillium lilacinum cepa 251 sobre $M$. incognita mostró resultados desfavorables; sin embargo, la combinación de fluopyram con P. lilacinum dio como resultado un nivel de control superior en comparación con su forma individual (Dahlin et al. 2019).

El objetivo de la presente investigación fue evaluar el efecto ejercido por tres productos bionematicidas formulados a base de Purpureocillium lilacinum y por el nematicida químico fluensulfone para el manejo de Nacobbus aberrans y Meloidogyne incognita en plantas de tomate cv. Río Grande.

\section{Materiales y Métodos}

\section{Obtención de inoculo y material vegetal}

Se usó una población de $N$. aberrans (T85) aislada de plantas de tomate provenientes del municipio de Tanhuato, Michoacán, México (Cabrera-Hidalgo et al. 2015; Chavarro-Carrero et al. 2017) y $M$. incognita obtenido de plantas de Solanum lycopersicun provenientes de la localidad de Mesón Nuevo, Ixtapan de la Sal, Estado de México, México.

El incremento del inóculo fue en raíces de plantas de Solanum lycopersicum cv. Río Grande susceptible a los nematodos agalladores en estudio. Se utilizó como sustrato arena estéril en contenedores. Cuarenta y cinco días después de la inoculación con los nematodos se procedió a la extracción de huevos de raíces agalladas de cada población mediante la metodología de Vrain (1977). Los huevos se recolectaron en el tamiz de 500 mallas y se incubaron a $28^{\circ} \mathrm{C}$ en cajas de Petri con agua destilada estéril. Se recuperaron los juveniles que eclosionaron y se mantuvieron a $4^{\circ} \mathrm{C}$.

Semillas de $S$. lycopersicum cv. Río Grande se pusieron a germinar sobre papel absorbente estéril, humedecidas con agua destilada estéril, en cajas de 
plástico con tapa, incubadas a $27^{\circ} \mathrm{C}$. Catorce días después de la germinación, las plantas se trasplantaron en sustrato estéril turba más perlita, en una relación de mezcla (1:1), en vasos de poliestireno blanco de 473 $\mathrm{mL}$, y se dejaron crecer por cinco días.

\section{Inoculación de Nacobbus aberrans y Meloidogyne incognita}

Cinco días después del trasplante, la inoculación se llevó a cabo con 2,000 juveniles de segundo estadio (J2) y 1,000 huevos por plántula por tratamiento (T2 a T7), excepto en el control (Cuadro 1). La inoculación con $\mathrm{J} 2$ y huevos de manera independiente se hizo en $1 \mathrm{~mL}$ de agua destilada estéril, con el apoyo de una jeringa hipodérmica con aplicación directa al sustrato en la base del tallo de la planta.
Las plantas se mantuvieron en una cámara de crecimiento Thermo Scientific Modelo 845 (Thermo Fisher Scientific Inc., Asheville, NC. USA), bajo condiciones controladas de temperatura $25^{\circ} \mathrm{C}$, HR de $60 \%$ y horas luz/noche de 12/12 por 45 días a partir de la inoculación. Se aplicaron riegos cada dos días y dos fertilizaciones cada 10 días, con el producto Ultrasolmultiproposito $^{\circledR}$ 18-18-18 + ME, chileno (SQM Industrial S. A., Santiago de Chile), a una dosis de $1 \mathrm{~g}$ $\mathrm{L}^{-1}$.

\section{Diseño experimental}

El experimento se llevó a cabo bajo un diseño completamente al azar con siete tratamientos indicados en el Cuadro 1. La unidad experimental fue una planta con 10 repeticiones por tratamiento.

\begin{tabular}{|c|c|c|c|c|}
\hline $\begin{array}{l}\text { Tratamientos } \\
\text { (Tx) }\end{array}$ & $\begin{array}{l}\text { Nombre } \\
\text { comercial }\end{array}$ & $\begin{array}{l}\text { Concentración } \\
\text { UFC o i. a. } \mathrm{g}^{-1}\end{array}$ & Empresa & $\begin{array}{c}\text { Dosis } \\
\text { utilizada/Tx }\end{array}$ \\
\hline T1 Control & --- & --- & --- & --- \\
\hline $\begin{array}{l}\text { T2 Control inoculado con } \\
\text { Nacobbus aberrans }\end{array}$ & -- & --- & --- & --- \\
\hline $\begin{array}{l}\text { T3 Control inoculado con } \\
\text { Meloidogyne incognita }\end{array}$ & --- & --- & --- & --- \\
\hline T4 Purpureocillium lilacinum & Nemaroot $^{\circledR}$ & $1 \times 10^{8} \mathrm{UFC} \mathrm{g}^{-1}$ & $\begin{array}{l}\text { INNOVAK } \\
\text { GLOBAL }\end{array}$ & $3.5 \mathrm{~g} \mathrm{~L}^{-1}$ \\
\hline T5 Purpureocillium lilacinum & BioAct $^{\circledR}$ Prime & 4.7x1010 UFC $\mathrm{g}^{-1}$ & BAYER & $0.75 \mathrm{~mL}^{-1}$ \\
\hline T6 Purpureocillium lilacinum & Nematicida PI ${ }^{\circledR}$ & $1 \times 10^{11} \mathrm{UFC} \mathrm{g}^{-1}$ & SOLENA & $0.5 \mathrm{~g} \mathrm{~L}^{-1}$ \\
\hline T7 Fluensulfone $40.08 \%$ CE & Nimitz 480EC ${ }^{\circledast}$ & $480 \mathrm{~g}$ de i. $\mathrm{a} \mathrm{L}^{-1}$ & ADAMA & $200 \mu \mathrm{L}^{-1}$ \\
\hline
\end{tabular}

\section{Aplicación de tratamientos}

Veinticuatro horas después de la inoculación con $N$. aberrans y $M$. incognita se procedió a la aplicación de los tratamientos (Tx). Las dosis y forma de aplicación de los Tx se hicieron de acuerdo con las recomendaciones del fabricante de cada producto, según se detalla en el Cuadro 1. Para los tratamientos T4 a T6 se tomó la dosis correspondiente y se preparó un volumen de suspensión de conidios e inmediatamente se aplicaron $40 \mathrm{~mL}$ a cada plántula del respectivo tratamiento. El tratamiento con el nematicida fluensulfone (T7) se efectuó en una sola aplicación al sustrato 10 días antes del trasplante y de la inoculación con los nematodos.

\section{Variables evaluadas y análisis estadístico}

Cuarenta y cinco días después de la aplicación de los tratamientos, se evaluó el índice de agallamiento, así como el número de masas de huevos por planta y el número de huevos por gramo de raíz, además de la altura de plantas, el peso fresco de los tallos más hojas (peso aéreo) y el peso fresco de raíz (Chavarro-Carrero et al. 2017).

El índice de agallamiento (IA) se determinó con base en la escala de Taylor y Sasser (1978) (Cuadro 2) y se transformó a porcentaje mediante la fórmula: $\mathrm{PA}=[\Sigma($ n.v $) / \mathrm{N} . \mathrm{C}] 100 ;$ donde $\mathrm{PA}=$ porcentaje de agallamiento, $\mathrm{n}=$ número de raíces evaluadas en 
cada categoría, $\mathrm{v}$ = valor numérico de cada categoría, $\mathrm{N}=$ número total de raíces evaluadas y $\mathrm{C}=$ categoría mayor (Townsed y Heuberger 1943). Con estos datos se calculó la efectividad biológica de cada producto mediante la fórmula: $\mathrm{PC}=(\mathrm{X}-\mathrm{Y} / \mathrm{X}) \times 100$; donde $\mathrm{PC}=$ Porcentaje de control, $\mathrm{X}=$ Porcentaje de agallamiento en el testigo, y $\mathrm{Y}=$ Porcentaje de agallamiento en el tratamiento (Abbott 1925).

\begin{tabular}{|c|c|}
\hline Clase & Número de agallas o masas de huevos \\
\hline 0 & Plantas sin agallas o sin masas de huevos \\
\hline 1 & Plantas con 1 o 2 agallas o masas de huevos \\
\hline 2 & $\begin{array}{l}\text { Plantas con entre } 3 \text { y } 10 \text { agallas o masas de } \\
\text { huevos }\end{array}$ \\
\hline 3 & $\begin{array}{l}\text { Plantas con entre } 11 \text { y } 30 \text { agallas o masas de } \\
\text { huevos }\end{array}$ \\
\hline 4 & $\begin{array}{l}\text { Plantas con entre } 31 \text { y } 100 \text { agallas o masas de } \\
\text { huevos }\end{array}$ \\
\hline 5 & $\begin{array}{l}\text { Plantas con más de } 100 \text { agallas o masas de } \\
\text { huevos }\end{array}$ \\
\hline
\end{tabular}

Para determinar el número de masas de huevos en raíz (NMH) se contaron éstas en cada una de las plantas inoculadas; para ello, las raíces se sumergieron en una solución de Floxina B Sigma-Aldrich $\left[0.15 \mathrm{~g} \mathrm{~L}^{-1}\right.$ de agua] (Merck KGaA, Darmstadt, Alemania), durante $20 \mathrm{~min}$, para que las masas de huevos se tiñeran de rojo y se facilitara el conteo. Para determinar el número de huevos por gramo de raíz (NHG), se hizo la extracción en un gramo de raíz siguiendo la metodología de Vrain (1977) y se contabilizó el número de huevos.

La altura de las plantas (AP) se midió con un flexómetro; la medida se tomó desde la base hasta el ápice del tallo principal. El peso fresco aéreo (PFA) se estimó a partir de los tallos y hojas que se cortaron desde la base hasta el ápice del tallo de las plantas; el peso fresco de raíz (PFR) se obtuvo de todo el sistema radical al que previamente se le removió el sustrato. El material vegetal se pesó en una balanza analítica Ohaus modelo CS 200 (Ohaus Corporation, Parsippany, NJ USA).

Se comprobó la homogeneidad de varianza entre tratamientos mediante la prueba de Chi cuadrada y la distribución normal de las observaciones de cada tratamiento por la prueba de Shapiro Wilk, con niveles de significancia de $\mathrm{P}<0.05$. Posteriormente, los datos de cada variable se sometieron a un análisis de varianza y a una prueba de diferencia mínima significativa (DMS) $(p=0.05)$, con el paquete InfoStat Software Estadístico (2020). Se calculó el coeficiente de correlación de Pearson entre concentración de UFC g-1 de los bionematicídas y las variables IA y AP.

\section{Resultados Y Discusión}

\section{Índice de agallamiento (IA)}

Las plantas de S. lycopersicum cv. Río Grande inoculadas con N. aberrans y M. incognita y sin tratar presentaron un IA promedio de $81 \%$ y $73 \%$, respectivamente. En los tratamientos con el hongo $P$. lilacinus, el IA se dio en un rango de 8 a $19 \%$ para $N$. aberrans, y de 7 a $17 \%$ para M. incognita; esto representó una correlación negativa con la concentración de esporas presentes en cada tratamiento, según la correlación de Pearson

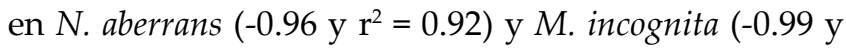
$\left.\mathrm{r}^{2}=0.98\right)$, lo cual significó que conforme aumentó la concentración de esporas, el IA disminuyó. Así, a la concentración de $1 \times 10^{11}$ UFC g-1 se registró el menor IA, y el mayor lo presentó el tratamiento con $1 \times 10^{8}$ UFC $\mathrm{g}^{-1}$ (Cuadro 3). La reducción del IA obtenido en el presente trabajo en $M$. incognita fue de 90.4 y $76.7 \%$ en los tratamientos T6 y T4, respectivamente, datos superiores a los reportados por otros autores. Freitas et al. (1995) encontraron una disminución de 37\% en plantas de tomate $\mathrm{cv}$. Rutgers inoculadas con $M$. javanica y tratadas con $P$. lilacinus; en otro estudio, al inocular P. lilacinus cepa 251 sobre $M$. incognita en plantas de tomate, se reportó una reducción de $66 \%$ en el IA (Kiewnick y Sikora 2006); con la misma cepa (cepa 251), a dosis de $0.2 \mathrm{~g}$ en $100 \mathrm{cc}$ de suelo, el IA disminuyó 3.2\% y 61\% de juveniles J2 (Dahlin et al. 2019). Contrastante es el resultado que se reporta en este trabajo con la cepa 521 (BioAct ${ }^{\circledast}$ Prime, Bayer), al reducir $84.9 \%$ el IA en relación con el control inoculado. El nematicida fluensulfone ( $2 \mathrm{~L} \mathrm{ha}^{-1}$ ) redujo el IA a $61.7 \%$ en $N$. aberrans y $65.7 \%$ en $M$. incognita (Cuadro 3). En un estudio similar, efectuado por Cabrera-Hidalgo et al. (2015), se obtuvo una reducción de $80 \%$ en el IA en plantas de S. lycopersicum cv. Río Grande, inoculadas con $N$. aberrans y tratadas con fluensulfone a dosis de $2 \mathrm{~L} \mathrm{ha}^{-1}$. 
Cuadro 3. Efecto de los tratamientos sobre el índice de agallamiento, número de masas de huevos y número de huevos por gramo de raíz de N. aberrans y M. incognita en plantas de tomate cv. Río Grande

\begin{tabular}{|c|c|c|c|c|c|c|}
\hline \multirow{2}{*}{ Trat. } & \multicolumn{3}{|c|}{ Nacobbus aberrans } & \multicolumn{3}{|c|}{ Meloidogyne incognita } \\
\hline & IA $(\%)$ & $\mathrm{NMH}$ & NHG & IA $(\%)$ & $\mathrm{NMH}$ & NHG \\
\hline T1 & 0 & $0^{\mathrm{a}}$ & $0^{\mathrm{a}}$ & 0 & $0^{\mathrm{a}}$ & $0^{\mathrm{a}}$ \\
\hline $\mathrm{T} 2$ & 81 & $122^{\mathrm{f}} \pm 4.3$ & $922.5^{\mathrm{f}} \pm 30.5$ & --- & --- & --- \\
\hline T3 & --- & --- & --- & 73 & $92^{\mathrm{e}} \pm 4.4$ & $637^{e} \pm 23.4$ \\
\hline $\mathrm{T} 4$ & 19 & $24^{\mathrm{d}} \pm 2.3$ & $209^{d} \pm 16.7$ & 17 & $21^{\mathrm{d}} \pm 1.2$ & $141.5^{\mathrm{d}} \pm 11.1$ \\
\hline T5 & 14 & $19^{c} \pm 1.4$ & $112.5^{c} \pm 6.7$ & 11 & $15.5^{c} \pm 1.3$ & $100^{c} \pm 5.0$ \\
\hline T6 & 8 & $5^{b} \pm 0.6$ & $31^{\mathrm{b}} \pm 4.1$ & 7 & $4.5^{\mathrm{b}} \pm 0.6$ & $15.5^{\mathrm{b}} \pm 1.4$ \\
\hline T7 & 31 & $29^{\mathrm{e}} \pm 2.1$ & $291^{\mathrm{e}} \pm 16.6$ & 25 & $13.5^{\mathrm{c}} \pm 1.1$ & $92^{c} \pm 3.0$ \\
\hline
\end{tabular}

Los datos IA, NMH y NHG son representativos de dos réplicas del experimento. Literales diferentes en columnas representan diferencias significativas mediante la prueba DMS ( $\mathrm{p}=0.05) \pm$ error estándar. $\mathrm{T} 1$ = plantas de tomate $\sin$ inocular y sin tratamiento (control); $\mathbf{T} 2=$ plantas inoculadas con $N$. aberrans y sin tratar; T3 = plantas inoculadas con $M$. incognita y sin tratar; $\mathrm{T} 4=P$. lilacinus $\left(1 \times 10^{8} \mathrm{UFC} \mathrm{g-1}\right) ; \mathrm{T} 5=P$. lilacinus $\left(4.7 \times 10^{10} \mathrm{UFC}^{-1}\right) ; \mathrm{T} 6=P$. lilacinus $\left(1 \times 10^{11}\right.$

$\mathrm{UFC} \mathrm{g}^{-1}$ ); T7 = fluensulfone; $\mathrm{IA}$ = índice de agallamiento; NMH = número de masas de huevos; NHG = número de huevos por gramo de raíz.

\section{Número de masas de huevos (NMH).}

Hubo diferencias significativas entre tratamientos en ambos nematodos. Purpureocillium. lilacinus en la concentración de $1 \times 10^{11} \mathrm{UFC} \mathrm{g}^{-1}$ redujo a 5 masas de huevos de $N$. Aberrans, las cuales contrastan con 122 en las plantas inoculadas sin tratar, y con 4.5 masas de huevos por planta en M. incognita contra 92 en el tratamiento T3. El efecto de la aplicación de los tratamientos se vio reflejado en la reducción del $\mathrm{NMH}$, al contrastarlos con el testigo inoculado; el tratamiento con P. lilacinum (Nematicida PI ${ }^{\circledR} 1 \times 10^{11}$ UFC g-1 Solena), a dosis de $0.5 \mathrm{~g}$ $\mathrm{L}^{-1}$, registró la reducción más alta del NMH: 95.9\% en $N$. aberrans y $95.1 \%$ en $M$. incognita; estos valores decrecieron en los tratamientos de menor concentración de UFC $\mathrm{g}^{-1}$ (Cuadro 3). Estudios similares indican que $P$. lilacinus cepa 251 logró reducir a 74\% el NMH con población final de nematodos en la raíz de $71 \%$, en plantas de tomate infestadas con M. incognita (Kiewnick y Sikora 2006); resultados próximos son reportados por Dahlin et al. (2019), con $62.8 \%$ en la reducción del número de masa de huevos con la aplicación de BioAct WG ( $P$. lilacinum cepa 251), a dosis de $0.2 \mathrm{~g}$ en $100 \mathrm{cc}$ de suelo; mientras que los resultados en este trabajo muestran una reducción de $83.2 \%$ del NMH en $M$. incognita inoculados con P. lilacinum (BioAct ${ }^{\circledR}$ Prime $1 \times 10^{10} \mathrm{UFC} \mathrm{g}^{-1}$ Bayer), en relación con el control inoculado. En los tratamientos con fluensulfone se encontraron en promedio 29 masas de huevos de $N$. aberrans, y un número menor para $M$. incognita (3.5); estos valores significan una reducción del NMH de 76.2 y 85.3\%, respectivamente (Cuadro 3). Lo anterior es atribuible posiblemente a las acciones pleiotrópicas de fluensulfone, al inhibir el desarrollo, la puesta y eclosión de huevos, la alimentación y la locomoción de larvas (Kearn et al. 2014), una reducción aceptable si se considera que el nematicida fluopyram presentó una reducción de 83\% del NMH de M. incognita (Dahlin et al. 2019).

\section{Número de huevos por gramo de raíz (NHG)}

Se tuvo un efecto significativo $(P=0.0001)$ en la reducción de NHG en $N$. aberrans y $M$. incognita por efecto de los tratamientos. En las plantas de tomate cv. Río Grande inoculadas con N. aberrans y tratadas con el producto Nematicida PI $\left(1 \times 10^{11} \mathrm{UFC}^{-1}\right)$ en promedio se registraron 31 huevos por g de raíz, contra 922.5 en plantas inoculadas sin tratar; en este mismo orden, el NHG en M. incognita fue de 15.5 contra 637 en el tratamiento T3. La reducción del NHG en los tratamientos se vio influenciada por la concentración de UFC $\mathrm{g}^{-1}$ declarada en cada producto evaluado, tal y como quedó demostrado en este trabajo; por ejemplo, el tratamiento T6 $\left(1 \times 10^{11} \mathrm{UFC}^{-1}\right)$ presentó una reducción de $96.6 \%$ en $N$. aberrans y de $97.6 \%$ en $M$. incognita, mientras que en el tratamiento T4 $\left(1 \times 10^{8}\right.$ $\mathrm{UFC}^{-1}$ ) el NHG fue de $77.3 \%$ en N. aberrans y de $77.8 \%$ en $M$. incognita. La reducción del número de huevos de ambos nematodos tratados con $P$. lilacinus puede ser atribuible a la acción parasítica directa del estadio sedentario de los nematodos, en particular el estadio de huevo, aunque la producción de leucinotoxinas, quitinasas, proteasas y ácido acético también han sido asociados en su proceso de infección (Djian et 
al. 1991; Khan et al. 2003, 2004; Park et al. 2004). En particular, la producción de quitinasas y proteasas ha sido reportado como el modo de acción de P. lilacinus cepa 251 contenida en el producto BioAct ${ }^{\circledR}$ Prime de Bayer (tratamiento T5 de este estudio); mediante este mecanismo, el hongo es capaz de infectar cualquier estadio de $M$. incognita, al degradar la cutícula del nematodo y el coreón de los huevos (Cayrol et al. 1989); esto puede explicar la formación baja de huevos en los tratamientos. Con el nematicida fluensulfone la reducción del NHG fue de $68.4 \%$ en N. aberrans y de $85.6 \%$ en $M$. incognita, en relación con los respectivos testigos inoculados (Cuadro 3); dentro de las formas de acción de esta molécula diferente al resto de nematicidas convencionales está la inhibición de puesta de huevos (Kearn et al. 2014), característica que queda evidenciada con los resultados obtenidos en la presente investigación.

\section{Efectividad biológica (EB)}

Los bajos valores del IA en los tratamientos con $P$. lilacinus se reflejaron en la EB. Los valores de efectividad biológica establecidos en la modificación a la Norma Oficial Mexicana NOM-032-FITO-1995, señalan que los productos a base de microorganismos deben manifestar valores iguales o mayores a $50 \%$, mientras que para los nematicidas químicos al menos
80\% (SAGARPA 2015); los porcentajes alcanzados por los bionematicidas evaluados en la presente investigación fluctuaron en un rango de 76.6 a 92.4\%, valores por encima de los establecidos oficialmente; resaltan los valores de EB obtenidos en el tratamiento T6 (Nematicida PI ${ }^{\circledR} 1 \times 10^{11} \mathrm{UFC} \mathrm{g}^{-1}$ Solena), con $90.1 \%$ en $N$. aberrans y $92.4 \%$ en $M$. incognita. Respecto al nematicida fluensulfune, la EB alcanzó $61.7 \%$ para $N$. aberrans y $65.5 \%$ para $M$. incognita, a dosis de $200 \mu \mathrm{l}$ $\mathrm{L}^{-1}$ (0.2 ppm) (Figura 1). Cabrera-Hidalgo et al. (2015) reportaron in vitro una mortalidad de $100 \%$ de J2 de $N$. aberrans a una concentración de 288 ppm.

\section{Altura de las plantas (AP)}

Los síntomas y lesiones en plantas causadas por nematodos han sido caracterizados en diferentes trabajos; dichas respuestas se asocian con los hábitos parasíticosquepresentanlosfitonematodosagalladores. En general, la AP se redujo cuando éstas fueron inoculadas con $N$. aberrans y $M$. incognita. Las plantas tratadas con $P$. lilacinus a una concentración de $1 \times 10^{11}$ $\mathrm{UFC}^{-1}$ presentaron una reducción promedio de $24.6 \%$ con $N$. aberrans, mientras que las plantas inoculadas y sin tratar se redujeron en un porcentaje de $51.8 \%$ con relación al control; con $M$. incognita la reducción fue de $2.9 \%$, mientras que en plantas inoculadas y sin tratar fue de $44.3 \%$; esto marcó diferencias significativas

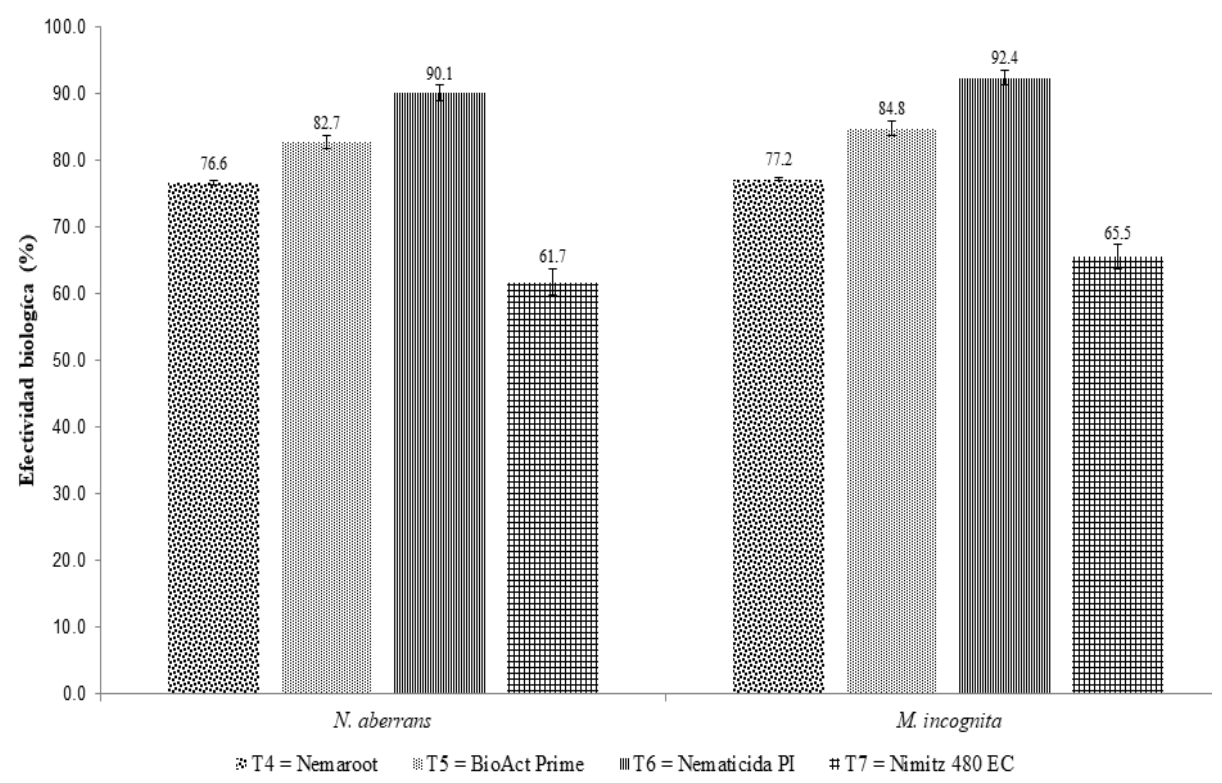

Figura 1. Efectividad biológica de tratamientos en el control de N. aberrans y M. incognita en plantas de tomate cv. Río Grande. Los datos son representativos de dos experimentos efectuados en ocasiones separadas. No se incluyen los tratamientos 1 a 3 debido a que la efectividad biológica fue 0 . 
entre tratamientos $(P=0.0001)$ (Cuadro 4$)$. La variable AP se correlacionó positivamente con la concentración de esporas $\left(0.88\right.$ y r $\left.{ }^{2}=0.77\right)$, lo que significó que cuando la concentración de esporas incrementó, la altura de la planta aumentó. Los tratamientos con fluensulfone manifestaron una reducción de AP de $47.5 \%$ con $N$. aberrans y con M. incognita tan solo de $8.9 \%$ con respecto a las plantas control. De acuerdo con Chavarro-Carrero et al. (2017), las alteraciones morfológicas y estructurales en el sistema radical se encuentran estrechamente relacionadas con la reducción en la altura de la planta y su peso, ya que limita o bloquea la translocación de agua y nutrientes estadística $(P=0.0001)$. Las plantas tratadas con el producto Nematicida PI $\left(1 \times 10^{11}\right.$ esporas $\left.\mathrm{g}^{-1}\right)$ e inoculadas con $N$. aberrans redujeron PFA en $18.6 \%$; pero fue el tratamiento de $M$. incognita el que presentó la menor reducción, con $7.3 \%$, comparados con las plantas control (T1). En este orden, los tratamientos con el nematicida Nimitz 480 EC $^{\circledR}$ tuvieron una reducción de PFA de 29.1 y $25.8 \%$ (Cuadro 4). En contraste, en plantas de pepino inoculadas con $M$. incognita y tratadas con P. lilacinus más Syncephalastrum racemosum Cohn, se incrementó la longitud de brote, el peso fresco y el rendimiento, en relación con el control (Huang et al. 2016).

Cuadro 4. Efecto de tratamientos sobre altura de planta, peso fresco aéreo y peso fresco de raíz de planta de tomate inoculadas con N. aberrans y M. incognita.

\begin{tabular}{|c|c|c|c|c|c|c|}
\hline \multirow{2}{*}{ Trat. } & \multicolumn{3}{|c|}{ Nacobbus aberrans } & \multicolumn{3}{|c|}{ Meloidogyne incognita } \\
\hline & $\mathrm{AP}(\mathrm{cm})$ & PFA (g) & PFR (g) & $\mathrm{AP}(\mathrm{cm})$ & PFA (g) & PFR (g) \\
\hline T1 & $40.50^{f} \pm 0.76$ & $11.85^{\mathrm{e}} \pm 0.26$ & $7.46^{\mathrm{d}} \pm 0.18$ & $31.50^{\mathrm{e}} \pm 0.13$ & $15.10^{\mathrm{e}} \pm 0.14$ & $12.90^{\mathrm{e}} \pm 0.16$ \\
\hline $\mathrm{T} 2$ & $19.53^{\mathrm{a}} \pm 0.52$ & $3.42^{\mathrm{a}} \pm 0.17$ & $1.66^{\mathrm{ab}} \pm 0.16$ & --- & --- & --- \\
\hline T3 & -- & -- & --- & $17.55^{\mathrm{a}} \pm 0.17$ & $9.40^{\mathrm{a}} \pm 0.09$ & $5.20^{\mathrm{a}} \pm 0.05$ \\
\hline $\mathrm{T} 4$ & $24.37^{c} \pm 0.33$ & $5.10^{\mathrm{b}} \pm 0.12$ & $1.48^{a} \pm 0.07$ & $26.28^{b} \pm 0.26$ & $10.70^{\mathrm{b}} \pm 0.19$ & $9.45^{b} \pm 0.15$ \\
\hline T5 & $26.01^{\mathrm{d}} \pm 0.43$ & $5.25^{\mathrm{b}} \pm 0.12$ & $1.90^{\mathrm{b}} \pm 0.04$ & $28.70^{c} \pm 0.25$ & $11.50^{c} \pm 0.12$ & $11.05^{\mathrm{c}} \pm 0.14$ \\
\hline T6 & $30.52^{\mathrm{e}} \pm 0.31$ & $9.64^{\mathrm{d}} \pm 0.11$ & $2.35^{c} \pm 0.04$ & $30.60^{\mathrm{d}} \pm 0.34$ & $14.00^{\mathrm{d}} \pm 0.11$ & $12.35^{\mathrm{d}} \pm 0.17$ \\
\hline T7 & $21.23^{\mathrm{b}} \pm 0.34$ & $8.40^{c} \pm 0.15$ & $1.60^{\mathrm{a}} \pm 0.08$ & $28.68^{c} \pm 0.23$ & $11.20^{c} \pm 0.17$ & $10.85^{\mathrm{c}} \pm 0.17$ \\
\hline
\end{tabular}

Los datos AP, PFA y PFR son representativos de dos réplicas del experimento. Literales diferentes en columnas representan diferencias significativas mediante prueba DMS ( $\mathrm{p}=0.05) \pm$ error estándar. T1 = plantas de tomate sin inocular y sin tratamiento (control); $\mathbf{T} 2=$ plantas inoculadas con $N$. aberrans y sin tratar; T3 = plantas inoculadas con $M$. incognita $\mathrm{y} \sin$ tratar; $\mathrm{T} 4=P$. lilacinus $\left(1 \times 10^{8} \mathrm{UFC} \mathrm{g}^{-1}\right) ; \mathrm{T} 5=P$. lilacinus $\left(4.7 \times 10^{10} \mathrm{UFC} \mathrm{g}^{-1}\right)$; T6 $=P$. lilacinus $\left(1 \times 10^{11}\right.$ $\left.\mathrm{UFC}^{-1}\right) ; \mathrm{T} 7$ = fluensulfone; $\mathrm{AP}=$ altura de planta; $\mathrm{PFA}=$ peso fresco aéreo; $\mathrm{PFR}=$ peso fresco de raíz.

necesarios para su crecimiento. Nacobbus aberrans es el único fitonematodo capaz de salir del hospedante y reingresar a éste, presentar una etapa migratoria endoparasítica y una etapa sedentaria (Eves-van den Akker et al. 2014), lo que implica modificaciones en la estructura celular (Kyndt et al. 2013). Por el contrario, M. incognita, una vez que ingresa el juvenil J2, hace el resto de su ciclo biológico dentro de la raíz; en esta etapa sedentaria induce cambios en la estructura celular de la raíz y la formación de agallas (Kyndt et al. 2013), y, por consecuencia, una reducción en las actividades fisiológicas para limitar la altura de la planta.

\section{Peso fresco aéreo (PFA)}

Las plantas inoculadas con $N$. aberrans o $M$. incognita $\mathrm{y}$ tratadas con $P$. lilacinus manifestaron diferencia

\section{Peso fresco de raíz (PFR)}

El ANDEVA para la variable PFR mostró diferencias significativas entre tratamientos $(\mathrm{P}=0.0001)$. Las plantas inoculadas con $N$. aberrans y tratadas con el producto Nematicida PI manifestaron el mayor PFR (12.35 g), seguido en orden descendente conforme decreció la concentración de esporas por gramo en cada bionematicida (Cuadro 4); estos resultados se reflejaron en la reducción del PFR en los tratamientos T4, T5 y T6 con $80.2 \%, 74.5 \%$ y $68.5 \%$, respectivamente; y fluensulfone de $78.6 \%$ comparados con el control. Para el caso de $M$. incognita, el mayor PFR (12.35 g) se obtuvo con P. lilacinum $1 \times 10^{11}$ esporas $\mathrm{g}^{-1}$, esto representó una reducción de $4.3 \%$ de PFR con relación al control; los valores de esta variable estuvieron influenciados por la concentración de esporas declaradas en cada producto comercial (Cuadro 4). El bajo peso fresco de raíz indica 
su limitado desarrollo, lo cual está relacionado con la deformación (hipertrofia e hiperplasia) que ocurre en las células de la raíz. Huang et al. (2016) señalan que la aplicación de $P$. lilacinus más Syncephalastrum racemosum para el manejo de $M$. incognita en plantas de pepino estimula significativamente la longitud de la raíz.

El nivel de reducción en las variables IA, NMH y NHG estuvo directamente relacionado con la concentración de esporas. Sin embargo, el origen de la cepa de $P$. lilacinus y el sustrato sobre el cual crece son factores que determinan la capacidad del agente biocontrolador (Gortari y Hours 2016).

\section{Conclusión}

Este estudio revela que el hongo nematófago Purpureocillium lilacinum tiene una alta capacidad para reducir el índice de agallamiento y el número de masas de huevos de los nematodos N. aberrans y M. incognita, por lo que representa un componente más para ser considerado en el manejo de estos nematodos.

Los niveles de efectividad biológica obtenidos con Purpureocillium lilacinum fueron superiores a los que establece la NOM-032-FITO-1995 para los productos biológicos; destacó el producto Nematicida $\mathrm{PI}^{\circledR} 1 \times 10^{11}$ UFC $\mathrm{g}^{-1}$.

Los valores de las variables que presentó el nematicida fluensulfone para el control de los nematodos $N$. aberrans y $M$. incognita fueron menores en relación con los de $P$. lilacinum; sin embargo, estas estrategias química y biológica integradas podrían convertirse en un componente importante para el control de estos nematodos parásitos de plantas.

\section{Agradecimientos}

Se agradece a las empresas ADAMA México e INNOVAK GLOBAL por la donación del producto para la realización del estudio. 


\section{LITERATURA CITADA}

Abbott WS. 1925. A method of computing the effectiveness of an insecticide. Journal of Economic Entomology 18: 265-267. https://doi.org/10.1093/jee/18.2.265a

Bayer CropScience. [internet]. 2020. La revolución nematicida. Folleto Velum Prime, Bayer AG. España. [cited 2020 Julio 31]. Disponible en: https://www.cropscience. bayer.es/ /media/Bayer\%20CropScience/CountrySpain-Internet/labels/Velum\%20Prime.ashx?force=1

Beeman AQ, Tylka GL. 2018. Assessing the effects of ILeVO and VOTiVO seed treatments on reproduction, hatching, motility, and root penetration of the soybean cyst nematode, Heterodera glycines. Plant Disease 102: 107113. https://doi.org/10.1094/PDIS-04-17-0585-RE

Cabrera AJ, Valdovinos G, Mora G, Rebollar A, Marbán N. 2014. Ocurrencia de Nacobbus aberrans en cultivos hortícolas del noroeste de Michoacán. Nematropica 44: 107-117.

Cabrera-Hidalgo AJ, Valadez-Moctezuma E, Marbán N. 2015. Efecto del fluensulfone sobre la movilidad in vitro, y la reproducción y agallamiento de Nacobbus aberrans en microparcelas. Nematropica 45: 59-71.

Cayrol J-C, Djian C, Pijarowski L. 1989. Study of the nematocidal properties of the culture filtrate of the nematophagous fungus Paecilomyces lilacinus. Revue de Nematology 12(4): 331-336.

Cepeda-Siller M, García-Calvario JM, Hernández-Juárez A, Ochoa-Fuentes YM, Garrido-Cruz F, Cerna-Chávez E, Dávila-Medina MD. 2018. Toxicidad de extractos de Carya illinoinensis (Fagales: Junglandaceae) contra Meloidogyne incognita (Tylenchida: Heteroderidae) en tomate. Ecosistemas y Recursos Agropecuarios 5(13): 143-148.

Chavarro-Carrero EA, Valdovinos-Ponce G, GómezRodríguez O, Nava-Díaz C, Aguilar-Rincón VH, Valadez-Moctezuma E. 2017. Respuesta de la línea 35-3 de chile tipo huacle (Capsicum annuum) a dos poblaciones de Nacobbus aberrans. Nematropica 47: 74-85.

Companioni B, Domínguez G, García R. 2019. Trichoderma: su potencial en el desarrollo sostenible de la agricultura. Biotecnología Vegetal 19(4): 237-248.

Dahlin P, Eder R, Consoli E, Krauss J, Kiewnick S. 2019. Integrated control of Meloidogyne incognita in tomatoes using fluopyram and Purpureocillium lilacinum strain 251. Crop Protection 124: 1-7. https://doi.org/10.1016/j. cropro.2019.104874
InfoStat Software Estadístico. [internet]. 2020. Centro de Transferencia InfoStat. Di Rienzo JA, Casanoves F, Balzarini MG, Gonzalez L, Tablada M, Robledo CW. [2020 Feb 09]. Disponible en: http://www.infostat.com. ar

Djian C, Pijarowski L, Ponchet M, Arpin N, Favre-Bonvin J. 1991. Acetic acid: A selective nematicidal metabolite from culture filtrates of Paecilomyces lilacinus (Thom) Samson and Trichoderma longibrachiatum Rifai. Nematologica 37: 101-112. https://doi.org/10.1163/187529291X00105

Eves-van den Akker S, Lilley CJ, Danchin EGJ, Rancurel C, Cock PJA, Urwin PE, Jones JT. 2014. The transcriptome of Nacobbus aberrans reveals insights into the evolution of sedentary endoparasitism in plant-parasitic nematodes. Genome Biology and Evolution 6: 2181-2194. https://doi. org/10.1093/gbe/evu171

Faske TR, Hurd K. 2015. Sensitivity of Meloidogyne incognita and Rotylenchulus reniformis to Fluopyram. Journal of Nematology 47(4): 316-321.

Freitas LG, Ferraz S, Muchovej JJ. 1995. Effectiveness of different isolates of Paecilomyces lilacinus and an isolate of Cylindrocarpon destructans on the control of Meloidogyne javanica. Nematropica 25: 109-115.

Gortari MC, Hours RA. 2016. Purpureocillium lilacinum LPSC \# 876: Producción de conidias en cultivos sobre sustratos sólidos y evaluación de su actividad sobre Nacobbus aberrans en plantas de tomate. Revista de la Facultad de Agronomía 115(2): 239-249.

Huang WK, Cui JK, Liu SM, Kong LA, Wu QS, Peng H, Hea WT, Sunb JH, Peng DL. 2016. Testing various biocontrol agents against the root-knot nematode (Meloidogyne incognita) in cucumber plants identifies a combination of Syncephalastrum racemosum and Paecilomyces lilacinus as being most effective. Biological Control 92: 31-37. http:// doi.org/10.1016/j.biocontrol.2015.09.008

Huang B, Li J, Wang Q, Guo M, Yan D, Fang W, Ren Z, Wang Q, Ouyang C, Li Y, Cao A. 2018. Effect of soil fumigants on degradation of abamectin and their combination synergistic effect to root-knot nematode. PLoS One 13: e0188245. https://doi.org/10.1371/journal.pone.0188245

Kearn J, Ludlow E, Dillon J, O'Connor V, Holden-Dye L. 2014. Fluensulfone is a nematicide with a mode of action distinct from anticholinesterases and macrocyclic lactones. Pesticide Biochemistry and Physiology 109: 44-57. https://doi.org/10.1016/j.pestbp.2014.01.004

Khan A, Williams K, Molloy MP, Nevalainen H. 2003. Purification and characterization of a serine protease 
and chitinases from Paecilomyces lilacinus and detection of chitinase activity on 2D gels. Protein Expression and Purification 32: 210-220. https://doi.org/10.1016/j. pep.2003.07.007

Khan A, Williams KL, Nevalainen HKM. 2004. Effects of Paecilomyces lilacinus protease and chitinase on the eggshell structures and hatching of Meloidogyne javanica juveniles. Biological Control 31: 346-352. https://doi. org/10.1016/j.biocontrol. 2004.07.011

Kiewnick S, Sikora RA. 2006. Biological control of the rootknot nematode Meloidogyne incognita by Paecilomyces lilacinus strain 251. Biological Control 38: 179-187. https:// doi.org/10.1016/j.biocontrol.2005.12.006

Kyndt T, Vieira P, Gheysen G, de Almeida-Engler J. 2013. Nematode feeding sites: Unique organs in plant roots. Planta 238: 807-818. https://doi.org/10.1007/ s00425-013-1923-z

Luangsa-Ard J, Houbraken J, Van Doorn T, Hong SB, Borman AM, Hywel-Jones NL, Samson RA. 2011. Purpureocillium, a new genus for the medically important Paecilomyces lilacinus. Fems Microbiology Letters 321(2): 141-149. https://doi.org/10.1111/j.1574-6968.2011.02322.x Núñez-Camargo MC, Carrión G, Núñez-Sánchez ÁE, López-Lima JD. 2012. Evaluación de la patogenicidad in vitro de Purpureocillium lilacinum sobre Globodera rostochiensis. Agroecosistemas Tropicales y Subtropicales 15: S126-S134.

Oka Y, Shuker S, Tkachi N. 2012. Systemic nematicidal activity of fluensulfone against the root-knot nematode Meloidogyne incognita on pepper. Pest Management Science 68: 268-275. https://doi.org/10.1002/ps.2256

Park J-O, Hargreaves JR, McConville EJ, Stirling GR, Ghisalberti EL, Sivasithamparam K. 2004. Production of leucinostatins and nematicidal activity of Australian isolates of Paecilomyces lilacinus (Thom) Samson. Letters in Applied Microbiology 38: 271-276. https://doi. org/10.1111/j.1472-765X.2004.01488.x

[SAGARPA] Secretaría de Agricultura, Ganadería, Desarrollo Rural, Pesca y Alimentación. [internet]. 2015. Modificación a la Norma Oficial Mexicana NOM-032FITO-1995. Por la que se establecen los requisitos y especificaciones fitosanitarios para la realización de estudios de efectividad biológica de plaguicidas agrícolas y su Dictamen Técnico. [cited 2020 Marzo 26]. Disponible en: http://www.dof.gob.mx/nota_detalle.php?codig $o=5403310$ \&fecha $=11 / 08 / 2015$

[SIAP] Servicio de Información Agroalimentaria y Pesquera. [internet]. 2020. Avances de siembras y cosechas. [cited 2020 Marzo 26]. Disponible en: http://infosiap.siap.gob. mx:8080/agricola_siap_gobmx/AvanceNacionalCultivo. do

Taylor AL, Sasser JN. 1978. Biology, Identification and Control of Root-knot Nematodes (Meloidogyne species). CABI. Raleigh, USA.

Townsed GR, Heuberger JW. 1943. Methods for estimating losses caused by diseases in fungicides experiments. The Plant Disease Report 27: 340-343.

Vrain TC. 1977. A technique for the collection of larvae of Meloidogyne spp. and a comparison of eggs and larvae as inoculum. Journal of Nematology 9: 249-251. 